\title{
miR-34c-5p mediates the cellular malignant behaviors of oral squamous cell carcinoma through targeted binding of TRIM29
}

\author{
Yuchen Shen ${ }^{1,2 \#}$, Changsheng Sun ${ }^{1,2 \#}$, Bowen Zhao ${ }^{1,2}$, Haobing Guo ${ }^{1,2}$, Jianhao Li ${ }^{1,2}$, Yanyun Xia ${ }^{1,2}$, \\ Miaomiao Liu ${ }^{1,2}$, Songlin Piao ${ }^{1,2}$, Wuliji Saiyin ${ }^{1,2}$ \\ ${ }^{1}$ Department of Oral and Maxillofacial Surgery, The First Affiliated Hospital of Harbin Medical University, Harbin, China; ${ }^{2}$ School of Stomatology, \\ Harbin Medical University, Harbin, China \\ Contributions: (I) Conception and design: Y Shen, C Sun, S Piao; (II) Administrative support: S Piao, W Saiyin; (III) Provision of study materials or \\ patients: B Zhao, H Guo, J Li; (IV) Collection and assembly of data: Y Shen, Y Xia, M Liu; (V) Data analysis and interpretation: Y Shen, C Sun, B \\ Zhao; (VI) Manuscript writing: All authors; (VII) Final approval of manuscript: All authors. \\ \#These authors contributed equally to this work and considered as co-first authors. \\ Correspondence to: Songlin Piao; Wuliji Saiyin. 143 Yiman Street, Nangang District, Harbin, China. \\ Email: piaosonglin_21@163.com; saiyinwuliji2021@163.com.
}

\begin{abstract}
Background: This investigation examined the effects of the microRNA miR-34c-5p on the proliferation, migration, and invasion of oral squamous cell carcinoma (OSCC) and the mechanisms involved.

Methods: The Gene Expression Omnibus (GEO) database was used to filter the chips, and the GEO2R software (https:/www.ncbi.nlm.nih.gov/geo/geo2r/) was used to analyze the microarray data (GSE28100 and GSE45238). Gene set enrichment analysis (GSEA) was used to study the relationship between the expression of miR-34c-5p and the distant metastasis and pathological grade of OSCC. The correlation between TRIM29 (tripartite motif containing 29) expression and the malignant clinical phenotype of OSCC was also examined. The mRNA and protein expression levels of miR-34c-5p and TRIM29 were measured by real time quantitative reverse transcription polymerase chain reaction (RT-qPCR) and Western blot analysis. The proliferation, migration, invasion and apoptosis of the human oral squamous carcinoma cell lines CAL27 and Tca8113 was assessed by performing cell-counting kit-8 (CCK-8) assays, colony formation assays, transwell tests, wound scratch tests and flow cytometry. Luciferase reporter assays were used to predict the relationship between miR-34c-5p and TRIM29. A xenograft nude model was established and used to evaluate the effect of miR-34c-5p on tumor growth in female BALB/c mice.
\end{abstract}

Results: The expression of miR-34c-5p was significantly correlated with the proliferation, migration, and metastasis of OSCC. Overexpression of miR-34c-5p promoted the proliferation, migration, and invasion of CAL-27 and Tca8113 cells, and suppressed their apoptosis. Inversely, low expression of miR-34c-5p suppressed the proliferation, migration, and invasion of CAL-27 and Tca8113 cells, and promoted their apoptosis. Overexpression of miR-34c-5p promoted tumor growth in the xenograft nude mice model. The expression of TRIM29 was related to malignant clinical phenotype of OSCC. Overexpression of TRIM29 inhibited the proliferation, migration and invasion of CAL-27 and Tca8113 cell, and induced their apoptosis. TRIM29 knockout had just the opposite effect. Importantly, miR-34c-5p binds to TRIM29 and inhibited TRIM29 expression.

Conclusions: MiR-34c-5p regulates the proliferation, migration, invasion, and apoptosis of OSCC through targeted binding of TRIM29. This may represent a novel therapeutic target for the treatment of patients with OSCC.

Keywords: Oral squamous cell carcinoma (OSCC); miR-34c-5p; tripartite motif-containing 29 (TRIM29); cellular malignant phenotype 
Submitted Aug 07, 2021. Accepted for publication Oct 21, 2021.

doi: $10.21037 / \mathrm{atm}-21-4679$

View this article at: https://dx.doi.org/10.21037/atm-21-4679

\section{Introduction}

Oral cancer is the most common malignant tumor of the head and neck. In 2018, there were more than 350,000 diagnoses worldwide and more than 175,000 related deaths (1). More than $90 \%$ of oral cancers are oral squamous cell carcinomas (OSCC) (2). Although the diagnosis and treatment of OSCC has improved, the prognosis is still very poor, with a 5 -year survival rate of about $50 \%$ (3), and this is largely related to the aggressiveness of OSCC and lymph node metastasis (4). In fact, more than $50 \%$ of patients present with advanced lymph node metastasis at the initial clinical examination $(5,6)$. The tumor size and lymph node metastasis in the TNM staging system are currently important predictors for evaluating the prognosis of patients (7-9). Due to the heterogeneity of OSCC and the complex mechanisms involved in metastasis, there is currently a lack of effective treatments to inhibit metastasis. Therefore, it is crucial to investigate the mechanisms of OSCC metastasis so as to develop targeted treatments for patients with OSCC.

The development and widespread application of microarray chip technology, especially gene chips, has produced a wealth of high-throughput biological data for scientific research. The Gene Expression Omnibus (GEO) database and The Cancer Genome Atlas (TCGA), which uses sequencing-based genome analysis to construct a detailed "atlas" of cancer genome changes, contain abundant sequencing data related to almost all tumor types and the data is updated in real time.

MicroRNAs (miRNAs) are small non-coding RNAs of $22-23$ nucleotides that are widely found in eukaryotes. It is often combined with the 3'-untranslated region (3'-UTR) in messenger RNAs (mRNAs) to regulate gene expression, thereby affecting cell differentiation, proliferation, invasion, and apoptosis, as well as other physiological and pathological processes (10). Studies have found that miRNAs can participate in the tumorigenesis and development of a variety of malignancies by regulating key genes (11). Indeed, miRNAs can act as tumor suppressor genes or oncogenes and have been shown to promote the proliferation and invasion of OSCC cells (12). miR$34 c-5 p$, a novel miRNA, has been found to be involved in the tumorigenesis and development of multiple tumors
$(13,14)$. For example, methylated miR34c-5p suppresses proliferation and metastasis via directly regulating the special AT-rich sequence-binding protein 2 (SATB2) in colorectal cancer (15). Nevertheless, the molecular regulatory mechanism of miR-34c-5p in OSCC is unclear. Increasingly, there is evidence to show that the miRNA expression profile of cancer cells can be used as effective biomarkers for the diagnosis and prognosis of patients with cancer.

Tripartite motif-containing 29 (TRIM29), also known as the ataxia group D complementary gene (ATDC), is a member of the TRIM protein family. It is located on the $11 \mathrm{q} 23$ of human chromosome 11 . Investigations have reported the ability of TRIM29 to positively and negatively regulate tumorigenesis (16). TRIM29 has been found to be up-regulated in cervical cancer (17), pancreatic cancer (18), and colorectal cancer (19), and is associated with tumor progression and poor prognosis. In contrast, it acts as a tumor suppressor in hepatocellular carcinoma (20) and breast cancer (21). The expression of TRIM29 is significantly related to tumor size, tumor histological grade, lymph node metastasis, and tumor invasion $(22,23)$. Therefore, TRIM29 is a promising target for tumor therapy. However, there is a paucity of evidence supporting the role of TRIM29 in OSCC.

This study is the first time to report the role of miR$34 c-5 p$ in OSCC progression and explores the regulatory mechanisms of the miR-34c-5p/TRIM29 axis involved in the tumorigenesis and cell malignant behaviors of OSCC. We present the following article in accordance with the ARRIVE reporting checklist (available at https://dx.doi. org/10.21037/atm-21-4679).

\section{Methods}

\section{Microarray analysis}

The Gene Expression Synthesis (GEO) database was used to select the relevant gene chip, and the GEO2R software (https://www.ncbi.nlm.nih.gov/geo/geo2r/) was used to analyze the microarray data (GSE28100 and GSE45238) $(24,25)$. The gene chips were screened according to the conditions of $\mathrm{P}<0.05$ and $\log$ fold change (logFC) greater than 1 . To obtain a more accurate selection, the screening 
conditions of $\mathrm{P}<0.05$ and $\log \mathrm{FC}$ greater than 1.5 were applied for the miRNA chips. The study was conducted in accordance with the Declaration of Helsinki (as revised in 2013).

\section{Gene set enrichment analysis (GSEA)}

GSEA was performed on the mRNA data of OSCC samples from the TCGA database $(26,27)$. The relationship between miR-34c-5p expression and the distant metastasis and pathological grade of OSCC, as well as the relationship between TRIM29 expression and the malignant clinical phenotype of OSCC was examined.

\section{Cell culture}

The oral squamous carcinoma cell lines CAL-27 and Tca8113 were obtained from the American Type Culture Collection. Cells were cultured in Roswell Park Memorial Institute (RPMI)-1640 (Gibco, NY, USA) medium with $10 \%$ exosome-free fetal bovine serum (FBS, HyClone, UT, USA) at $37^{\circ} \mathrm{C}$ in a tissue culture chamber with $95 \%$ oxygen $\left(\mathrm{O}_{2}\right)$ and $5 \%$ carbon dioxide $\left(\mathrm{CO}_{2}\right)$.

\section{Cell transfection}

The pcDNA plasmid containing miRNA-negative control (miR-NC), miR34c-5p mimic, TRIM29, wildtype TRIM29 (TRIM29-wt) 3'UTR, mutant TRIM29 (TRIM29-mut) 3'UTR, short hairpin RNA negative control (shRNA-NC), and shRNA containing TRIM29 \#1 and \#2 were obtained from GenePharma (Shanghai, China). CAL-27 and Tca8113 cells were transfected using Lipofectamine 3000 (Invitrogen, CA, USA) according to the manufacturer's instructions.

\section{Cell-counting kit-8 (CCK-8) assay}

Cell proliferation was assessed using the cell counting kit8 (CCK-8) assay (Dojindo, Japan) as per the manufacturer's instructions. Briefly, CAL-27 and Tca8113 cells $(100 \mu \mathrm{L})$ were plated into 96-well plates (Corning, NY, U.S.A.) and incubated at $37^{\circ} \mathrm{C}$. After 24 hours, $10 \mu \mathrm{L}$ CCK-8 solution was added to each well and incubated at $37^{\circ} \mathrm{C}$ for $1-4$ hours. The absorbance was measured at $450 \mathrm{~nm}$ with a microplate reader (Thermo Fisher Scientific, USA). Experiments were repeated three times.

\section{Colony formation assays}

To determine the colony formation ability, CAL-27 and Tca8113 cells were transfected with miR-NC, miR-34c5p mimic, shRNA-NC, shRNA-TRIM29 \#1, shRNATRIM29 2\#, and miR-34c-5p plus TRIM29. Cells were harvested, washed with phosphate buffered saline (PBS) and cultured in 6-well plates at 500 cells per well for 14 days. The medium was discarded and the cells were fixed with ethanol for 30 minutes followed by staining with $0.5 \%$ crystal violet. Finally, cells were rinsed with deionized water, allowed to dry, and visualized under light microscopy.

\section{Transwell assay}

The invasion ability of cells was assessed using Matrigelcovered Transwell chambers (Corning, NY, USA), according to the manufacturer's instructions. Briefly, $600 \mu \mathrm{L}$ of DMEM medium containing $10 \%$ FBS was added into 24-well plates. The Transwell chambers were placed into the 24-well plates. Cells were placed into the upper chamber at 150,000 cells per well per $100 \mu \mathrm{L}$. After incubation for 48 hours, the cells that had migrated were gently washed twice with $1 \times$ PBS and fixed in $5 \%$ glutaraldehyde for 5 minutes at room temperature. The cells were then stained using $0.1 \%$ crystal violet for 20 minutes. Cells were observed and counted using an optical microscope and photos were obtained. The experiment was repeated three times.

\section{Wound scratch assay}

Cells were cultured in 6-well plates in DMEM medium with $10 \%$ FBS. After reaching confluence, a small pipette tip was used to make a vertical scratch along the bottom of each well in the 6-well plate. Cells were then incubated at $37^{\circ} \mathrm{C}$ for 24 hours. Images were taken at 0 and 24 hours using an inverted optical microscope (Olympus, Japan). The Image J software was used to measure the width of the scratch area and to calculate the healing rate of the cell scratches. The experiment was repeated three times.

\section{Flow cytometry}

CAL-27 and Tca8113 cells were digested with trypsin (Abcam China, Shanghai, China) and immobilized overnight with $70 \%$ ethanol at $4{ }^{\circ} \mathrm{C} .10 \mathrm{mg} / \mathrm{mL}$ RNaseA (Beyotime, Shanghai, China) and propidium iodide (Beyotime, Shanghai, China) were supplemented for overnight at $4{ }^{\circ} \mathrm{C}$. 
According to the manufacturer's instructions, a Annexin V-FITC/PI Kit (hermo Fisher Scientific, USA) was used to examine early apoptosis of CAL-27 and Tca8113 cells, and the apoptotic rates were analyzed using a flow cytometry (Thermo Fisher Scientific, USA).

\section{starBase database analysis}

The starBase database is a widely-used open-source platform for studying the non-coding (nc)RNA interactions from CLIP-seq, degradome-seq, and RNA-RNA interactome data $(28,29)$. In this study, the starBase database was used to predict the binding of miR-34c-5p and TRIM29.

\section{Luciferase reporter assays}

The 3'-UTR of the TRIM29 wild-type (wt) gene was cloned into plasmids containing luciferase (pmirGLO). GeneArt $^{\mathrm{TM}}$ Site-Directed Mutagenesis System (Thermo Fisher Scientific, CA, USA) was used to produce the mutant TRIM29 gene reporter (TRIM29-mut). CAL-27 cells were seeded into $24-$ well plates at $4 \times 10^{4}$ cells $/ \mathrm{mL}$ and transfected with the TRIM29-wt or the TRIM29-mut, with or without the miR-34c-5p mimic, using Lipofectamine ${ }^{\mathrm{TM}}$ 3000 (Invitrogen, CA, USA). After 48 hours, the luciferase activity was measured with the Luciferase Reporter Assay System (Luciferase Assay Reagent, Promega, USA) on the Turner BioSystems Instrument as per the manufacturer's protocol (30).

\section{Real-time reverse transcription-polymerase chain reaction (qRT-PCR)}

Total RNA was extracted from CAL-27 and Tca8113 cells using TRIzol reagent (Invitrogen, Beijing, China) according to the manufacturer's instructions. The MiReasy Mini Kit Spin Column Kit (Qiagen Company, Germany) was used according to the manufacturers' protocol to obtain RNA with higher purity. The NanoDrop 2000 was used to check the concentration of the total RNA. Reverse transcription was performed using the PrimeScript RT reagent Kit (TakaRa, Dalian, China) as soon as possible to avoid RNA degradation. The 2 SYBR Premix Ex Taq ${ }^{\text {TM }}$ II (TakaRa, Dalian, China) was used to set up the qRTPCR reaction and the PCR was processed using the BioRad CFX-96 system (Bio-Rad, CA, USA). Glyceraldehyde3-phosphate dehydrogenase (GAPDH) or U6 was used as the normalization gene. The qRT-PCR data were analyzed using the $2^{-\Delta \Delta \mathrm{Ct}}$ method to calculate the relative expression of mRNA.

\section{Western blot assay}

Proteins were isolated from CAL-27 and Tca8113 cells using RIPA lysis buffer (Beyotime, Shanghai, China) according to the manufacturer's protocol. BCA Protein Assay Kit (Beyotime, Shanghai, China) was used to detect the protein concentrations. The proteins were separated using $10 \%$ dodecyl sulfate-polyacrylamide gel electrophoresis (SDS-PAGE) and transferred onto polyvinylidene difluoride (PVDF) membranes (Merck Millipore) by electro-blotting. Membranes were blocked with 5\% skim milk in Tris Buffered Saline Tween (TBST) for 1 hour at room temperature, followed by incubation with the primary rabbit anti-TRIM2 9 antibody overnight at $4{ }^{\circ} \mathrm{C}$. Membranes were then washed with TBST and incubated with the corresponding secondary antibodies conjugated to horseradish peroxidase (HRP) for 1 hour at room temperature. ECL reagent was used to visualize the protein bands and quantitative analysis was performed on the band intensities using an automatic digital gel image analysis system (Bio-Rad CFX-96, CA, USA). Beta-actin was used as the internal control to standardize sample loading.

\section{Nude mouse subcutaneous xenograft model}

All animal experiments were performed according to the National Institute of Health (NIH) Guide for the Care and Use of Laboratory Animals and were approved by the ethics committee of the First Affiliated Hospital of Harbin Medical University. A protocol was prepared before the study without registration. Female BALB/c nude mice aged 5-6 weeks old and weighing $18.31 \pm 5.63 \mathrm{~g}$ were purchased from the Department of Laboratory Animal Science of Harbin Medical University. A xenograft model was established by subcutaneous injection of $5 \times 10^{6}$ CAL- 27 cells $/ 0.1 \mathrm{~mL}$ into the right flank regions. Mice were randomly divided into two groups, namely, the miR-NC group that was injected with cells transfected with miR-NC, and the miR$34 c-5 p$ group that was injected with cells transfected with miR-34c-5p. After 1 week, successful formation of tumor was determine by tumor volume $>200 \mathrm{~mm}^{3}$. At 30 days post injection, mice were euthanized, and the tumors were excised and photographed. 


\section{Statistical analysis}

The endpoints, including differences in miRNA and mRNA expression, proliferation, migration, invasion, and luciferase assays, were assessed. Unpaired $t$-tests with Welch's correction were performed using GraphPad Prism 7.0 software. All statistical tests were two-sided, and $\mathrm{P}<0.05$ was considered statistically significant. Kaplan-Meier analysis was performed in GraphPad Prism 7.0 to determine the correlation between disease-free survival and TRIM29 expression.

\section{Results}

MiR-34c-5p is highly expressed in OSCC and is associated with the malignant clinical phenotype of OSCC

The GEO2R tool was used to analyze the expression of miR-34c-5p in OSCC samples in the GSE 45238 and GSE28100 databases. The results showed that the expression of miR-34c-5p in OSCC samples was 1.5 fold greater than that in healthy tissue, indicating that miR-34c$5 \mathrm{p}$ is high expression in OSCC tissues (Figure 1A). TCGA was used to study the relationship between the expression of miR-34c-5p and the distant metastasis and pathological grade of OSCC. The expression of $\mathrm{miR}-34 \mathrm{c}-5 \mathrm{p}$ was significantly higher in patients with M1 metastasis (where the cancer has spread to other parts of the body) compared to patients with M0 status (where cancer has not spread to other parts of the body) (Figure 1B). The expression of miR-34c-5p was also significantly higher in the G3-4 group (high grade cancer) compared to the G1-2 group (low and intermediate grade cancer) (Figure 1C), suggesting that the expression of miR-34c-5p is related to OSCC metastasis and pathological grading. OSCC patients were divided into two groups according to the median expression of miR-34c-5p, namely, the high expression group and the low expression group. GSEA analysis showed that the expression of miR-34c-5p was related to cell proliferation, migration, and metastasis (Figure 1D-1F).

\section{The effects of miR-34c-5p on cell proliferation, migration, invasion and apoptosis}

CAL-27 and Tca8113 cells were transfected with miR$\mathrm{NC}$ or miR-34c-5p mimic. RT-qPCR showed that the relative mRNA expression of miR-34c-5p was significantly increased in cells transfected with miR-34c-5p, confirming that the overexpression model was successfully constructed (Figure $2 A$ ). The CCK-8 assay and colony formation test demonstrated that miR-34c-5p overexpression promoted CAL-27 and Tca 8113 cell viability (Figure $2 B, 2 C$ ) and proliferation (Figure 2D). Furthermore, the wound scratch test revealed that overexpression of miR-34c-5p promoted the migration of CAL-27 and Tca8113 cells (Figure 2E,2F). Transwell assays demonstrated that miR-34c-5p overexpression induced CAL-27 and Tca8113 cell invasion (Figure 2G). Flow cytometry also indicated that miR-34c$5 \mathrm{p}$ overexpression suppressed apoptosis of CAL-27 and Tca8113 cells (Figure $2 H$ ).

Sponge RNAs contain complementary binding sites to the miRNA of interest and thus, were used to create miR$34 c-5 p$ loss of function in CAL-27 and Tca813 cells. RTqPCR showed that the relative mRNA expression of miR$34 c-5 p$ was significantly decreased in CAL-27 and Tca8113 cells transfected with sponge miR-34c-5p compared to cells transfected with sponge miR-NC (Figure 2I), indicating that the knockout model of miR-34c-5p was successfully constructed. Knock-down of miR-34c-5p in CAL-27 and Tca8113 cells resulted in reduced cell viability (Figure 27,2K), suppressed cell proliferation (Figure $2 L$ ), decreased cell migration ability (Figure $2 M, 2 N$ ), decreased cell invasion ability (Figure 2O), and increased apoptotic rate (Figure $2 P$ ). Taken together, these overexpression and silencing experiments suggested that miR-34c-5p promotes OSCC cell proliferation, migration, and invasion.

\section{Overexpression of miR-34c-5p promotes the growth of tumors in vivo}

A xenograft model was constructed by injecting nude mice with CAL-27 cells that had been transfected with miR-NC or miR-34c-5p. As shown in Figure 3A-3C, compared with miR-NC group, overexpression of miR-34c-5p significantly increased the size and volume of the transplanted tumor. Furthermore, overexpression of miR-34c-5p significantly increased the weight of the resultant tumor (Figure 3D). The mRNA expression of miR-34c-5p was markedly upregulated in tumors harvested from mice injected with miR-34c-5p CAL-27 cells compared to tumors from mice injected with miR-NC CAL-27 cells (Figure 3E). These results suggested that overexpression of miR-34c-5p promoted the growth of OSCC tumors in vivo.

\section{MiR-34c-5p binds to TRIM29 and inbibits TRIM29 expression}

Bioinformatic analyses (starBase 2.0) revealed a possible 


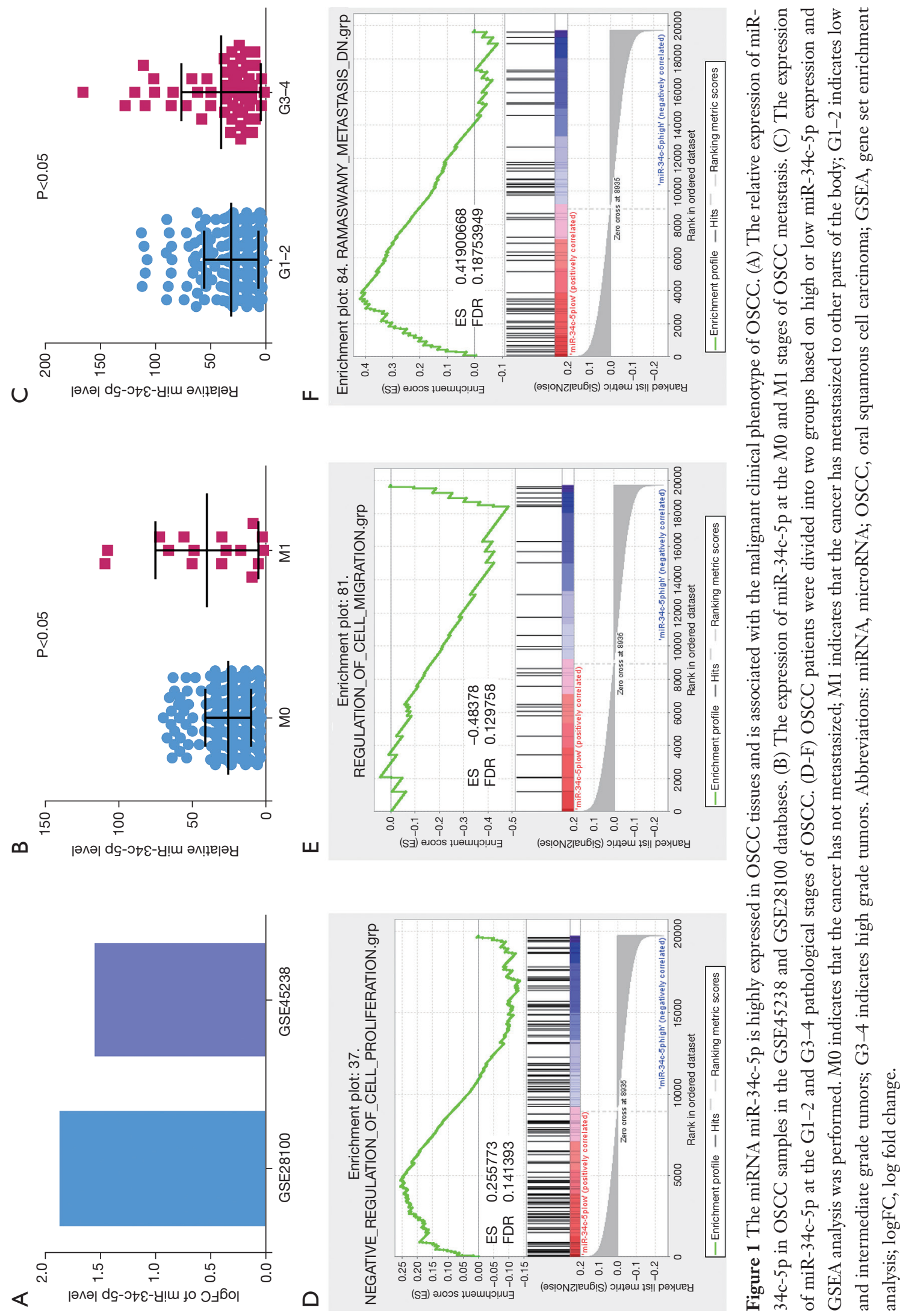



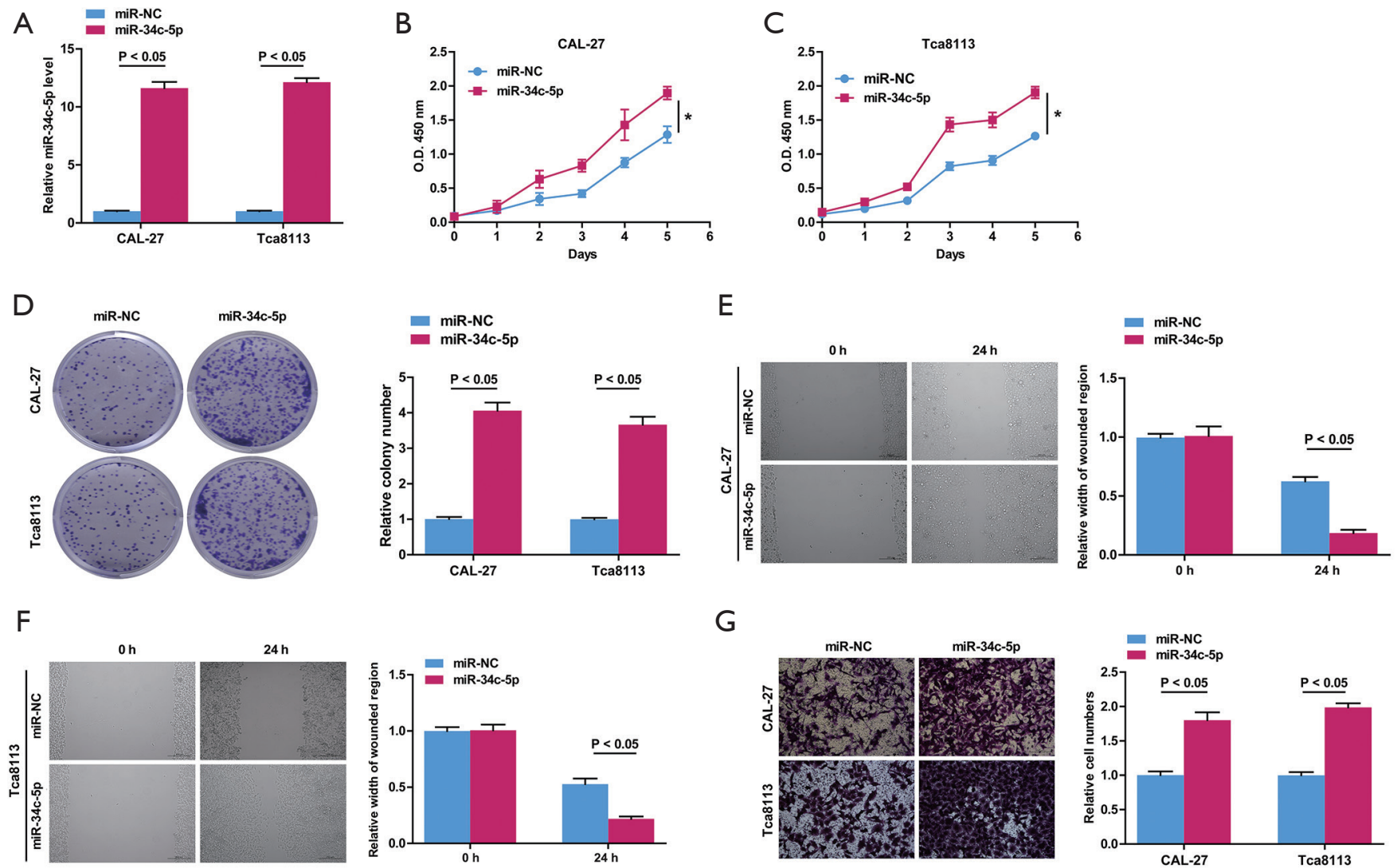

G
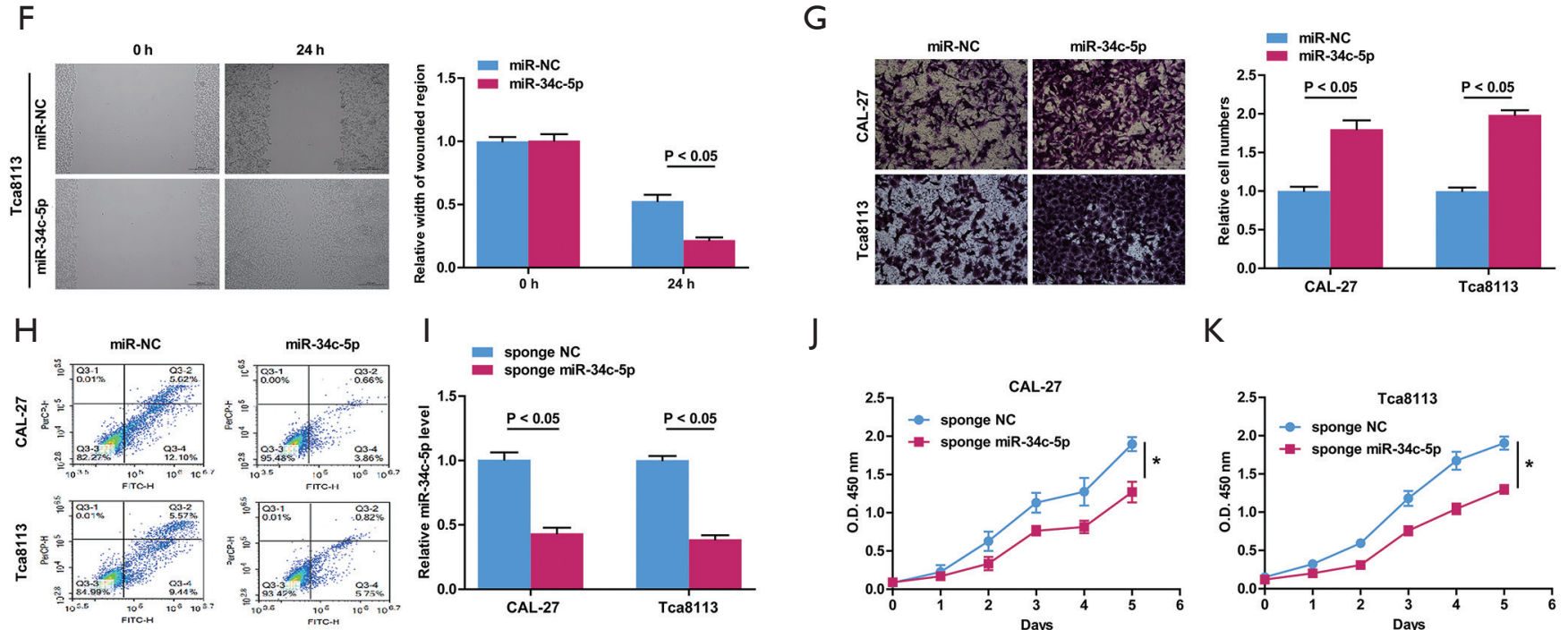

J

K
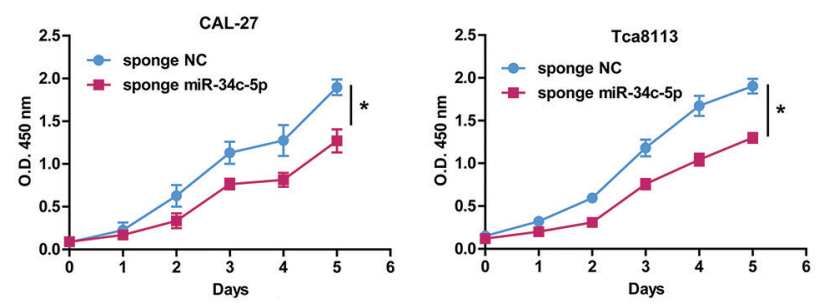

$\mathrm{L}$
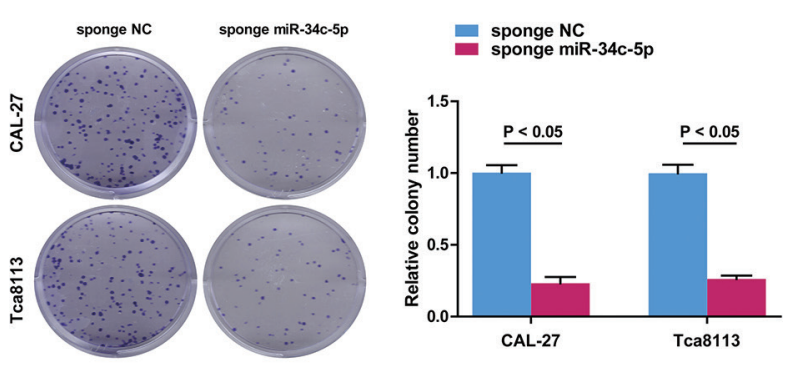

M
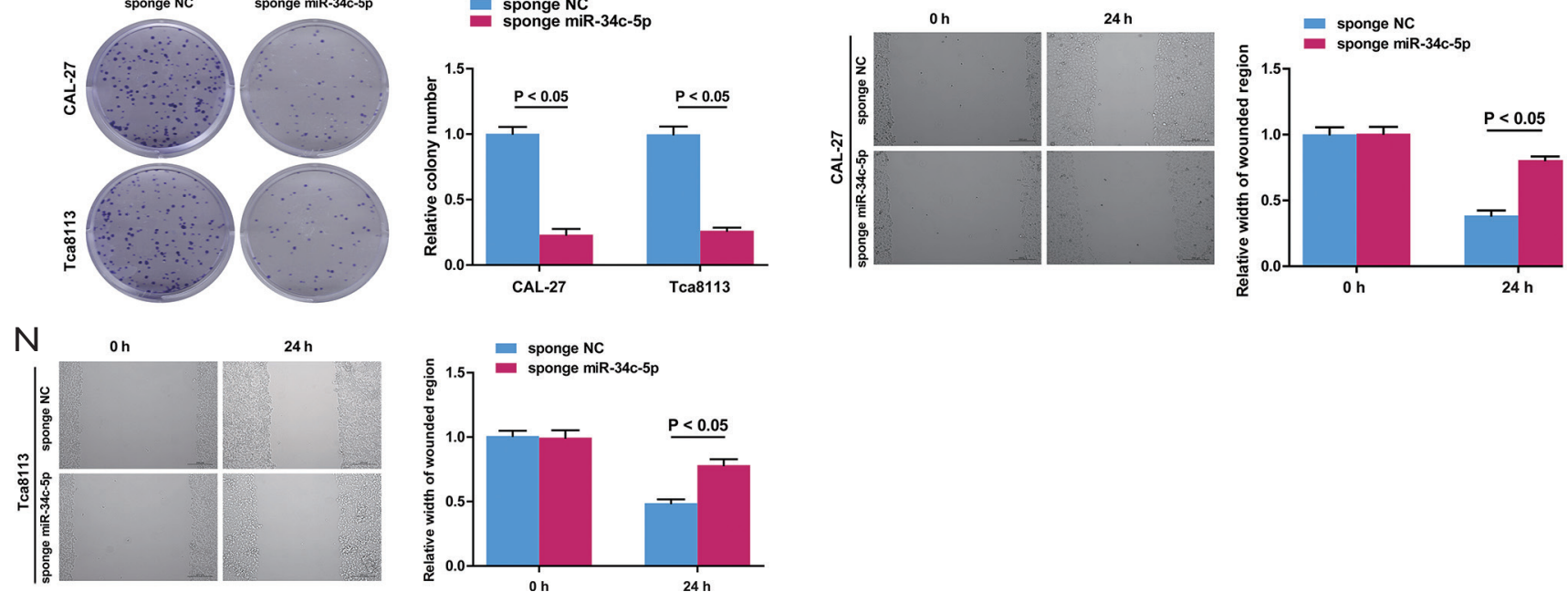

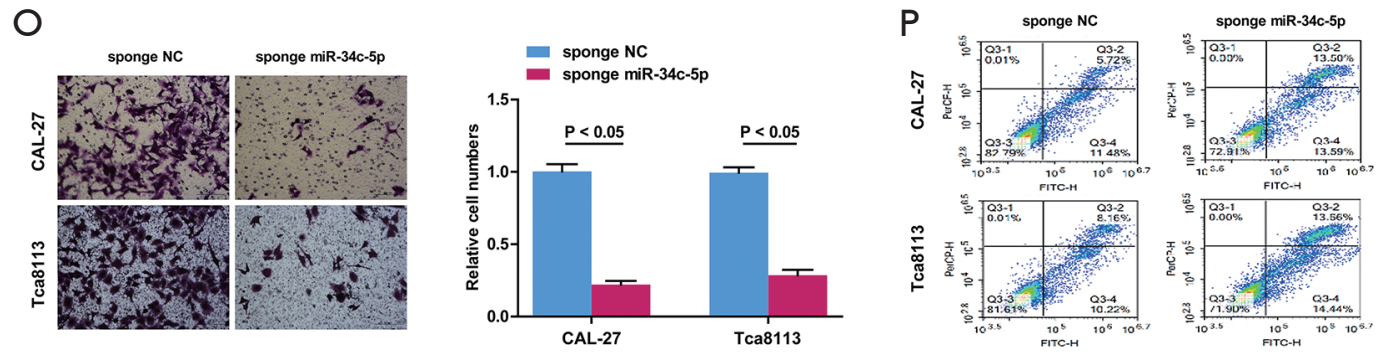

Figure 2 The effects of miR-34c-5p on OSCC cell proliferation, migration, invasion, and apoptosis. (A) RT-qPCR showing the relative mRNA expression of miR-34c-5p in CAL-27 and Tca8113 cells transfected with miR-NC or miR-34c-5p. (B) CCK-8 assay showing cell viability in CAL-27 cells transfected with miR-NC or miR-34c-5p ( ${ }^{*}, \mathrm{P}<0.05$ vs. miR-NC group). (C) CCK-8 assay showing cell viability in Tca8113 cells transfected with miR-NC or miR-34c-5p (*, $\mathrm{P}<0.05$ vs. miR-NC group). (D) Colony formation assays showing cell proliferation in CAL-27 and Tca8113 cells transfected with miR-NC or miR-34c-5p, a microscope with magnification of $100 \times$. (E) The wound scratch test showing cell migration ability in CAL-27 transfected with miR-NC or miR-34c-5p, scale bars $=200 \mu \mathrm{m}$. (F) The wound scratch test showing cell migration ability in Tca8113 cells transfected with miR-NC or miR-34c-5p, scale bars $=200 \mu \mathrm{m}$. $(\mathrm{G})$ Transwell test showing cell invasion ability in CAL-27 and Tca8113 cells transfected with miR-NC or miR-34c-5p, scale bars =200 $\mu \mathrm{m}$. (H) Flow cytometry showing apoptotic rate in CAL-27 and Tca8113 cells transfected with miR-NC or miR-34c-5p. (I) RT-qPCR showing the relative mRNA expression of miR-34c-5p in CAL-27 and Tca8113 cells transfected with sponge NC or sponge miR-34c-5p. (J) CCK8 assay showing cell viability in CAL-27 cells transfected with sponge $\mathrm{NC}$ or sponge miR-34c-5p (*, $\mathrm{P}<0.05$ vs. sponge $-\mathrm{NC}$ group). (K) CCK-8 assay showing cell viability in Tca8113 cells transfected with sponge $\mathrm{NC}$ or sponge miR-34c-5p (*, $\mathrm{P}<0.05$ vs. sponge $-\mathrm{NC}$ group). (L) Colony formation assays showing cell proliferation in CAL-27 and Tca8113 cells transfected with sponge NC or sponge miR-34c-5p, a microscope with magnification of 100x. (M) The wound scratch test showing cell migration ability in CAL-27 cells transfected with sponge NC or sponge miR-34c-5p, scale bars $=200 \mu \mathrm{m}$. (N) The wound scratch test showing cell migration ability in Tca8113 cells transfected with sponge NC or sponge miR-34c-5p, scale bars $=200 \mu \mathrm{m}$. (O) Transwell test showing cell invasion ability in CAL-27 and Tca8113 cells transfected with sponge NC or sponge miR-34c-5p, scale bars $=200 \mu \mathrm{m}$. (P) Flow cytometry showing apoptotic rate in CAL-27 and Tca8113 cells transfected with sponge NC or sponge miR-34c-5p. OSCC, oral squamous cell carcinoma; RT-qPCR, real time quantitative reverse transcription polymerase chain reaction; CCK-8, cell counting kit-8; NC, negative control; OD, optical density.

target relationship between miR-34c-5p and TRIM29 (Figure 4A). In CAL-27 cells co-transfected with the wild type (wt) TRIM29 3'-UTR and miR-34c-5p, luciferase activity was significantly inhibited compared to cells cotransfected with wt TRIM29 3'-UTR and miR-NC (Figure 4B). However, in cells co-transfected with the mutant (mut) TRIM29 3'-UTR, no significant effect on luciferase activity was observed (Figure $4 B$ ). Furthermore, RT-qPCR results showed that the relative mRNA expression of TRIM29 was markedly down-regulated in cells transfected with miR-34c-5p compared to cells transfected with miR-NC (Figure 4C). This was confirmed by Western blot results showing that the relative protein expression of TRIM29 was markedly down-regulated in miR-34c-5p cells compared to miR-NC cells (Figure 4D). These results suggested that miR-34c-5p binds to TRIM29, thereby inhibiting TRIM29 expression.

\section{TRIM29 is lowly expressed in OSCC and is associated with the malignant clinical phenotype of OSCC}

TCGA was used to study the relationship between the expression of TRIM29 and the malignant clinical phenotype of OSCC. The results showed that the expression of TRIM29 in OSCC tissues was significantly lower than that in healthy adjacent tissues (Figure 5A). Moreover, the expression of TRIM29 in stage I-III and stage IV samples was significantly lower than that observed in healthy adjacent tissues (Figure 5B). The expression of TRIM29 was lower in M1 OSCC samples compared to M0 samples (Figure 5C). The expression of TRIM29 in the N1 group was significantly lower than that in the N0 group (Figure 5D). The expression of TRIM29 in G3-4 group was significantly lower than that in G1-2 group (Figure 5E). Furthermore, the expression of TRIM29 was significantly lower in patients with recurring disease compared to 


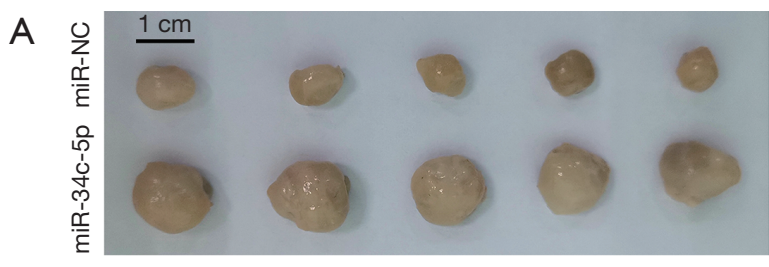

B

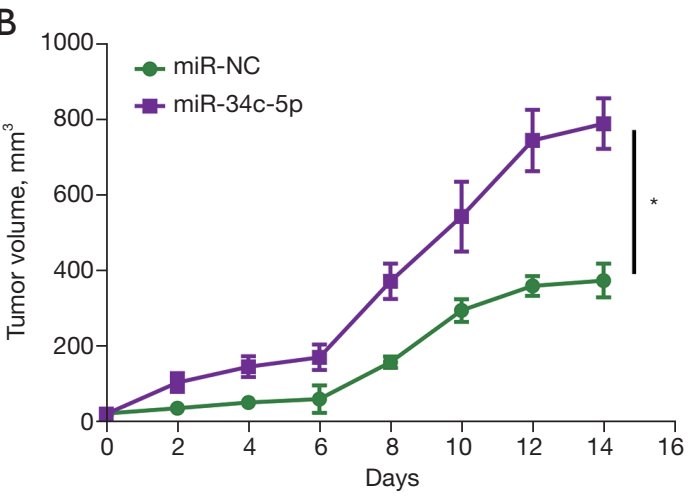

D

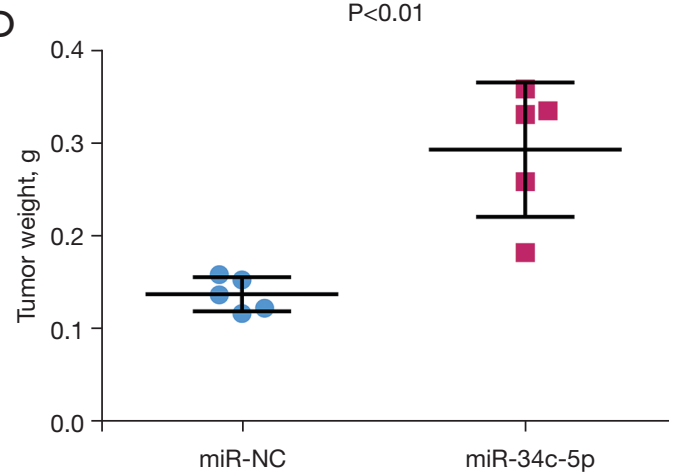

C

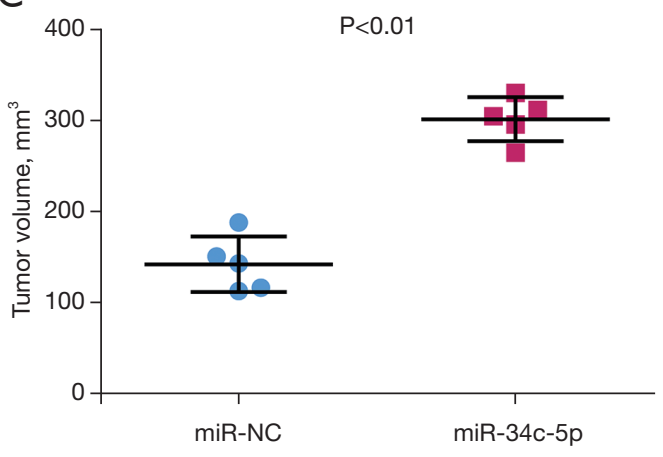

E

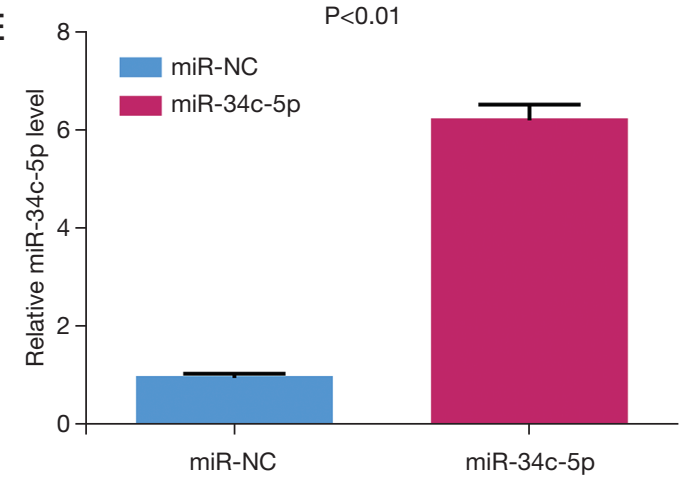

Figure 3 Overexpression of miR-34c-5p promotes the growth of transplanted tumors in vivo. (A) Tumors excised from nude mice at day 30 after subcutaneous injection of CAL-27 cells transfected with miR-NC or miR-34c-5p. (B) The tumor volume at days 2-14 after subcutaneous injection of CAL-27 cells ( ${ }^{*}, \mathrm{P}<0.05 v s$. miR-NC group). (C) The volume of the transplanted tumor at day 30 post-injection of CAL-27 cells. (D) The weight of the tumors on day 30 post-injection of CAL-27 cells. (E) The relative mRNA expression of miR-34c-5p in tumors from miR-NC mice and miR-24c-5p mice on day 30 post-injection of tumor cells. NC, negative control.

patients with no recurrence (Figure $5 F$ ). At the same time, the disease-free survival rate of patients with low TRIM29 expression was significantly poorer than that of patients with high TRIM29 expression (Figure $5 G$ ).

\section{The effects of TRIM29 on OSCC cell proliferation, migration, invasion and apoptosis}

CAL-27 and Tca8113 cells were transfected with TRIM29 or a vector control to examine the effects of TRIM29 overexpression on cell proliferation, migration, and invasion. As shown in Figure 6A, western blot analysis showed that the relative protein expression of TRIM29 was significantly highly in cells transfected with TRIM29 compared to cells transfected with the empty vector, indicating that the model overexpressing TRIM29 was successfully constructed. The CCK- 8 assay showed that TRIM29 overexpression inhibited cell viability (Figure 6B,6C). Overexpression of TRIM29 suppressed cell proliferation as shown by the colony formation assays (Figure 6D), and inhibited cell migration as shown by the wound scratch test (Figure 6E, $6 F$ ). Transwell assays revealed that cells overexpressing TRIM29 had reduced invasion ability compared to cells transfected with the 
A

'RIM29-WT: 5' aaAGGUGCCUUACACACUGCCc 3' miR-34c-5p: 3' cgUUAGUCGAUUGAUGUGACGGa 5' TRIM29-MT: 5' aaAGGUGCCUUACUGUGACGGc 3'

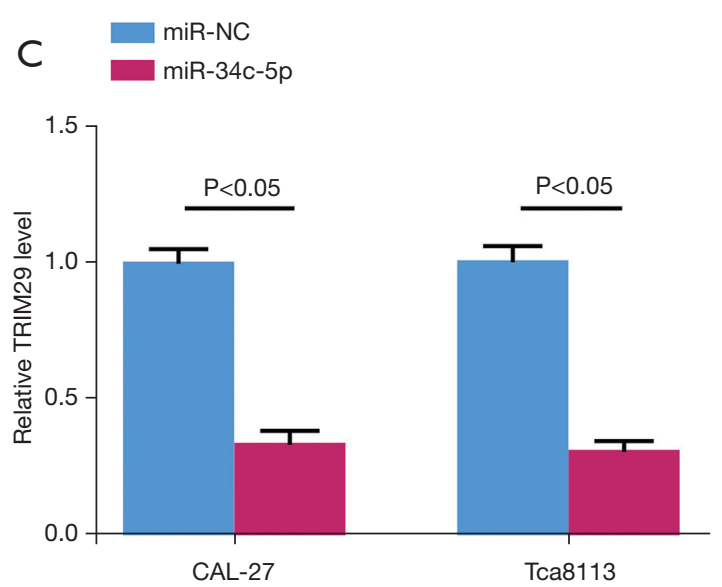

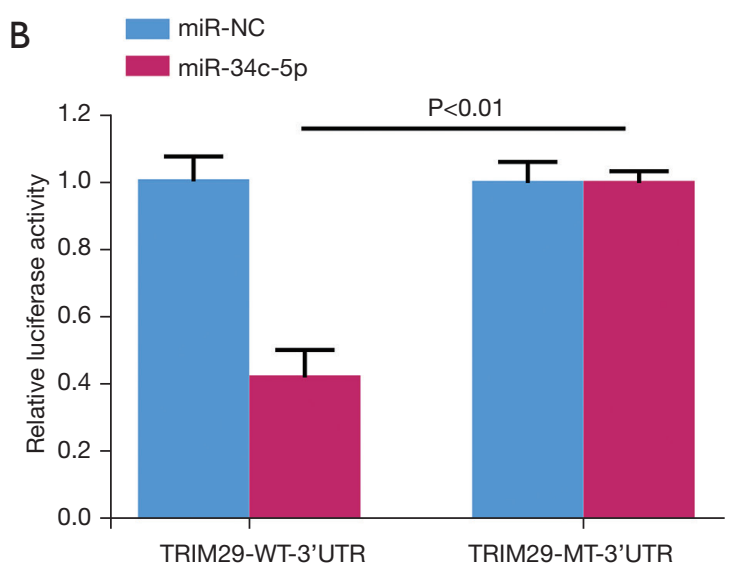

D

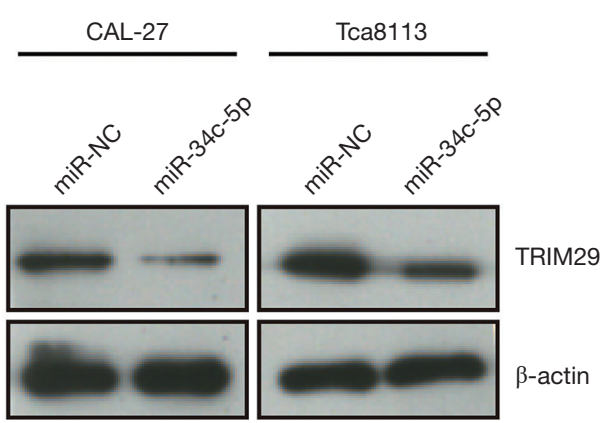

Figure 4 MiR-34c-5p binds to TRIM29 and inhibits TRIM29 expression. (A) starBase was used to predict the target relationship between miR-34c-5p and TRIM29. (B) Luciferase activity in CAL-27 cells transfected with wt or mut TRIM29 3'UTR and miR-NC or miR-34c5p. (C) RT-qPCR showing the relative mRNA expression of TRIM29 in CAL-27 and Tca8113 cells transfected with miR-NC or miR-34c5p. (D) Western blot showing the relative protein expression of TRIM29 in CAL-27 and Tca8113 cells transfected with miR-NC or miR34c-5p. TRIM29, tripartite motif-containing 29; wt, wildtype; mut, mutant; UTR, untranslated region; NC, negative control; RT-qPCR, real time quantitative reverse transcription polymerase chain reaction.

empty vector (Figure 6G). Flow cytometry demonstrated that overexpressing TRIM29 had elevated apoptotic rate compared to cells transfected with the empty vector (Figure 6H).

To silence TRIM29 expression, CAL-27 and Tca8113 cells were transfected with short hairpin RNA (sh-NC) or two different versions of shTRIM29 (\#1 and \#2). Western blot analysis showed that the relative protein expression of TRIM29 was significantly decreased in cells transfected with shTRIM29 compared to cells transfected with shNC, indicating that suppressed expression of TRIM29 was successfully achieved (Figure 6I). Cells in which TRIM29 expression was silenced (sh-TRIM29 \#1 and \#2) showed increased cells viability (Figure 67,6K), increased cell proliferation (Figure $6 L$ ), enhanced cell migration (Figure $6 \mathrm{M}, 6 \mathrm{~N}$ ), increased cell invasion ability (Figure 60 ), and inhibited apoptosis (Figure 6P). These results suggested that TRIM29 can affect OSCC cell proliferation, migration, invasion and apoptosis.

\section{MiR-34c-5p regulated OSCC cell proliferation, migration, invasion and apoptosis by targeting TRIM29}

Overexpression of miR-34c-5p in CAL-27 and Tca8113 cells significantly suppressed the protein expression of TRIM29. However, co-transfection of miR-34c-5p and TRIM29 (miR-34c-5p + TRIM29) alleviated this suppression and TRIM29 protein levels were comparable to that observed in cells transfected with miR-NC, and significantly higher than that observed in cells transfected with miR-34c-5p alone (Figure 7A). Co-expression of miR$34 c-5 p+$ TRIM29 reduced cell viability (Figure $7 B, 7 C$ ), cell 

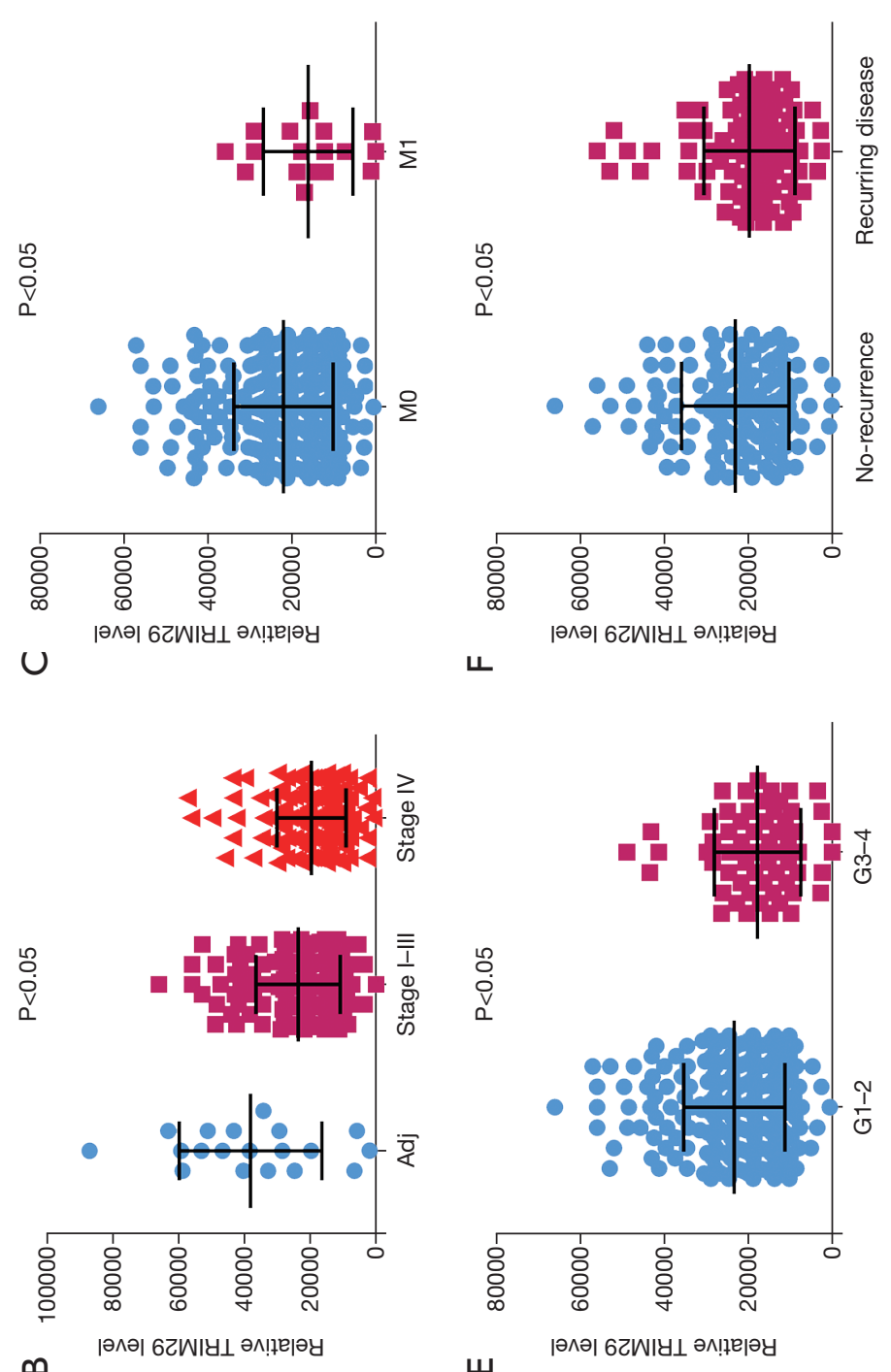

$\infty$

ш

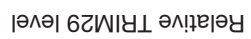

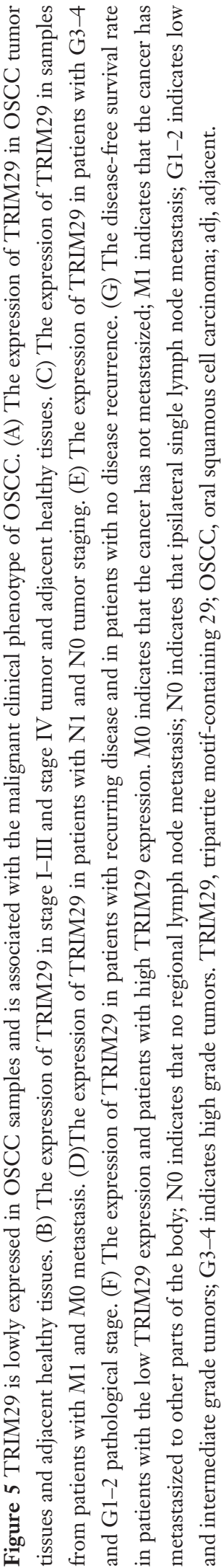


A

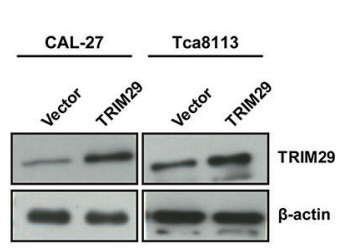

D

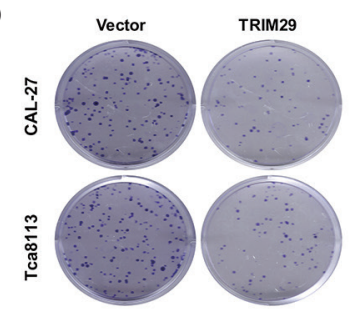

F

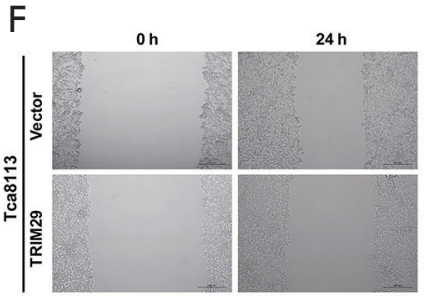

$\mathrm{H}$

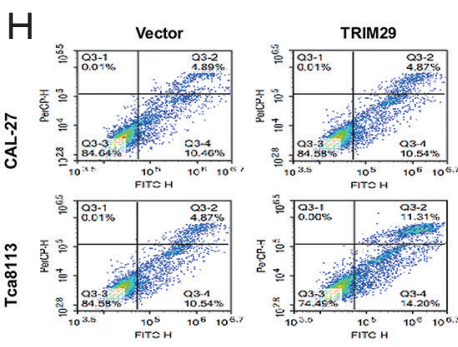

K

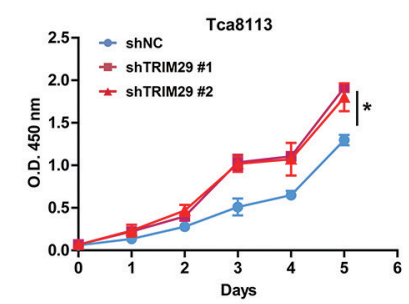

M

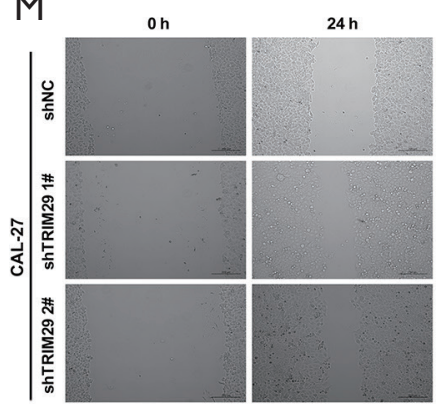

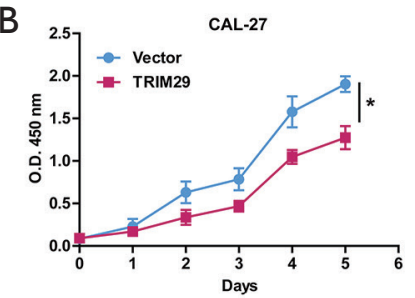
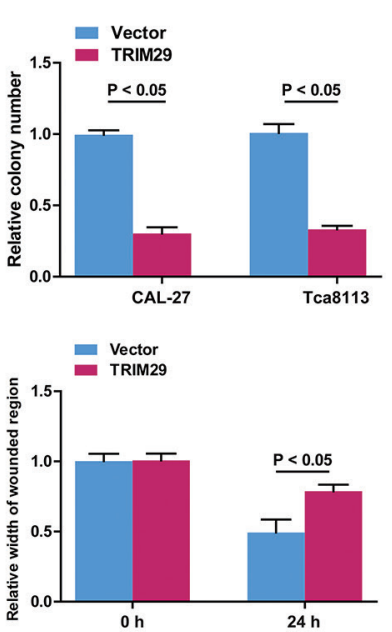

I

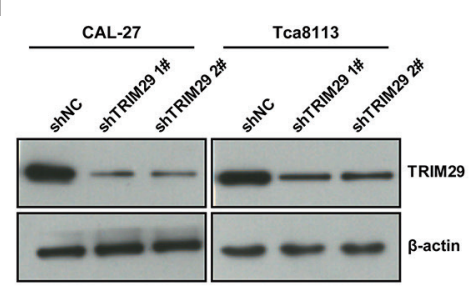

G

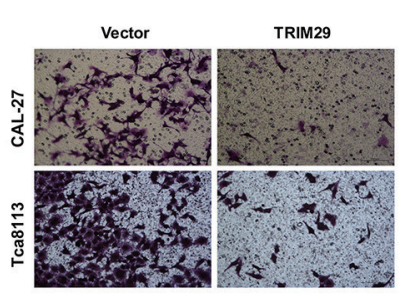

E
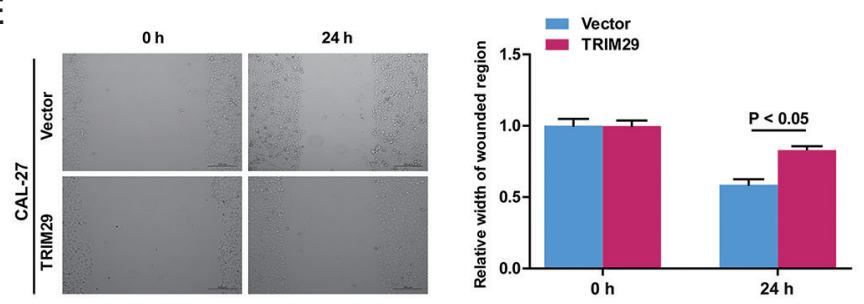

J

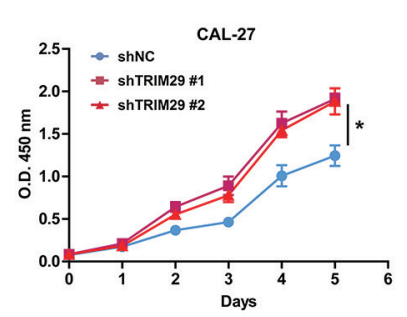

L
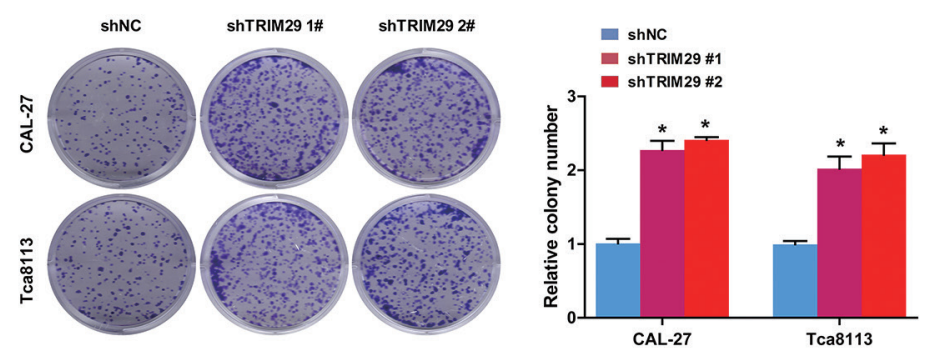

$\mathrm{N}$

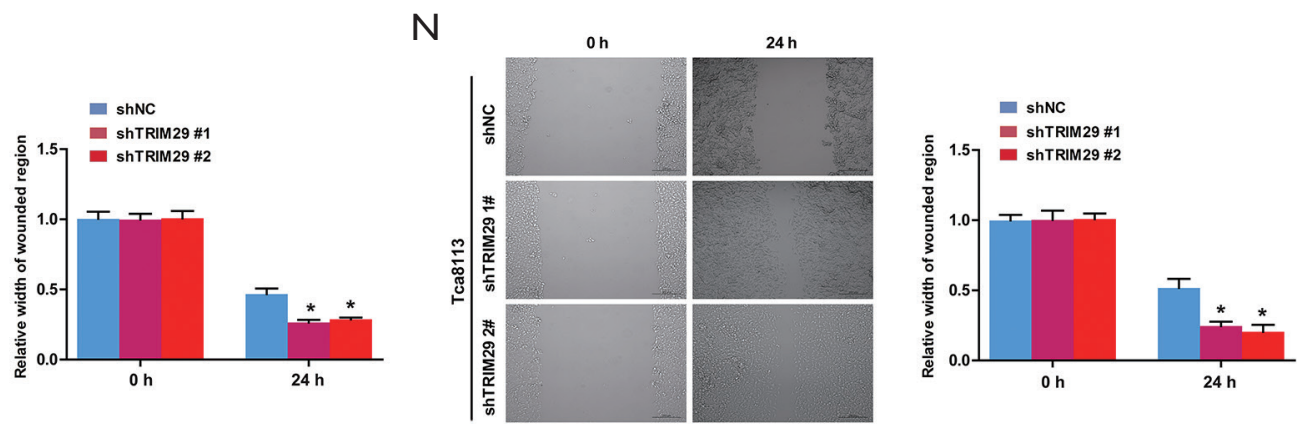

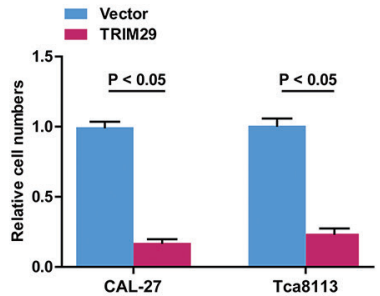

Tca8113 

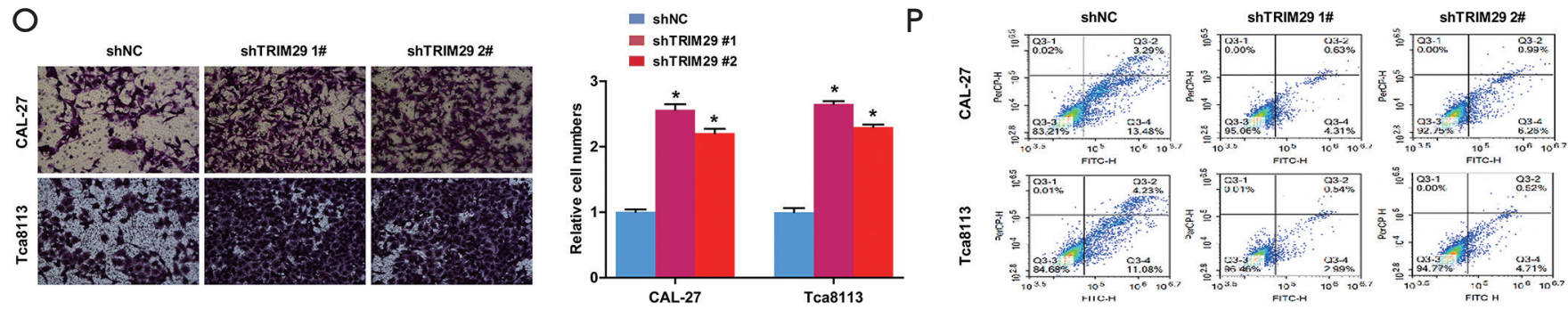

Figure 6 The effects of TRIM29 on OSCC cell proliferation, migration, invasion, and apoptosis. (A) Western blot showing the relative protein expression of TRIM29 in CAL-27 and Tca8113 cells transfected with null vector or vector containing TRIM29. (B) CCK-8 assay showing cell viability in CAL-27 cells transfected with null vector or TRIM29 ${ }^{*}, \mathrm{P}<0.05 v$ s. vector group). (C) CCK-8 assay showing cell viability in Tca8113 cells transfected with null vector or TRIM29 ( ${ }^{*}, \mathrm{P}<0.05$ vs. vector group). (D) The colony formation assay showing cell proliferation in CAL-27 and Tca8113 cells transfected with null vector or TRIM29, a microscope with magnification of $\times 100$. (E) The wound scratch test showing cell migration ability in CAL-27 cells transfected with null vector or TRIM29, scale bars $=200 \mu \mathrm{m}$. (F) The wound scratch test showing cell migration ability in Tca8113 cells transfected with null vector or TRIM29, scale bars $=200 \mu \mathrm{m}$. (G) Transwell test showing cell invasion ability in CAL-27 and Tca8113 cells transfected with null vector or TRIM29, scale bars =200 $\mu \mathrm{m}$. $(\mathrm{H})$ Flow cytometry showing apoptotic rate in CAL-27 and Tca8113 cells transfected with null vector or TRIM29. (I) Western blot showing the relative protein expression of TRIM29 in CAL-27 and Tca8113 cells silenced by transfection with shNC, sh-TRIM29 \#1, or sh-TRIM29 \#2. (J) CCK-8 assay showing cell viability in CAL-27 cells transfected with shNC, sh-TRIM29 \#1, or sh-TRIM29 \#2 ( ${ }^{*}, \mathrm{P}<0.05$ vs. shNC group). (K) CCK-8 assay showing cell viability in Tca8113 cells transfected with shNC, sh-TRIM29 \#1, or sh-TRIM29 \#2 $\left({ }^{*}, \mathrm{P}<0.05\right.$ vs. shNC group). (L) The colony formation assay showing cell proliferation in CAL-27 and Tca8113 cells transfected with shNC, sh-TRIM29 \#1, or sh-TRIM29 \#2 (*, $\mathrm{P}<0.05$ vs. shNC group), a microscope with magnification of 100×. (M) The wound scratch test showing cell migration ability in CAL-27 cells transfected with shNC, sh-TRIM29 \#1, or sh-TRIM29 \#2 (*, $\mathrm{P}<0.05$ vs. shNC group), scale bars $=200 \mu \mathrm{m}$. (N) The wound scratch test showing cell migration ability in Tca8113 cells transfected with shNC, sh-TRIM29 \#1, or sh-TRIM29 \#2 $\left({ }^{*}, \mathrm{P}<0.05\right.$ vs. shNC group), scale bars $=200 \mu \mathrm{m}$. (O) Transwell test showing cell invasion ability in CAL-27 and Tca8113 cells transfected with shNC, shTRIM29 \#1, or sh-TRIM29 \#2 (*, P $<0.05 v s . ~ s h N C$ group), scale bars $=200 \mu \mathrm{m}$. (P) Flow cytometry showing apoptotic rate in CAL-27 and Tca8113 cells transfected with shNC, sh-TRIM29 \#1, or sh-TRIM29 \#2 (*, $\mathrm{P}<0.05$ vs. shNC group). TRIM29, tripartite motif-containing 29; OSCC, oral squamous cell carcinoma; CCK-8, cell counting kit-8; sh, short hairpin; NC, negative control; OD, optical density.

proliferation (Figure $7 D$ ), cell migration (Figure $7 E, 7 F)$, cell invasion (Figure $7 G$ ), and inhibited apoptosis (Figure $7 H$ ) compared to cells overexpressing miR-34c-5p alone. These results suggested that miR-34c-5p promoted OSCC cell proliferation, migration and invasion, and suppressed apoptosis by regulating TRIM29 expression.

\section{Discussion}

OSCC is highly aggressive and readily metastasizes to regional lymph nodes. Patients often have a poor prognosis and low survival rates. Although the exact etiology of the disease is still unclear, the frontal cells of OSCC invasion play an important role in tumor invasion and metastasis. The progression of human cancer is due to an accumulation of genetic and epigenetic changes that can impair the function of signaling pathways (31). Multiple reports have suggested that miRNAs are important regulators of OSCC carcinogenesis $(14,32,33)$.

Generally, miRNAs can affect the growth, differentiation, and apoptosis of tumor cells by inhibiting the expression of multiple target genes (11). Interestingly, the same miRNA may act as a tumor-promoting gene or a tumor suppressor gene in different cancers. For example, miR$34 c-5 p$ inhibits the proliferation, migration, and invasion of osteosarcoma cells by targeting FLOT2 (34), and suppresses the metastasis and invasion of cervical cancer by targeting Notch 1 (35). However, miR-34c-5p can also promote colon cancer cell proliferation by targeting SIRT6 via activation of the JAK2/STAT3 signaling pathway (36).

In this study, bioinformatics analysis was performed on the GEO chip database to obtain the OSCC invasionrelated gene chip. Target gene prediction and functional annotation software was used identify tissue specific target genes. The miRNA miR-34c-5p was found to be highly expressed in OSCC tissues and its expression was 
A

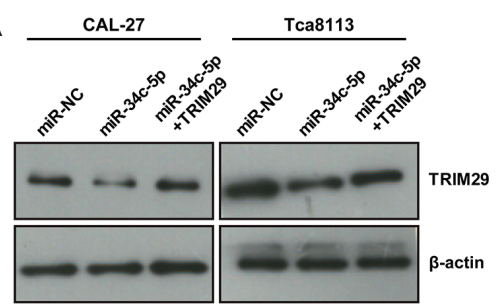

B

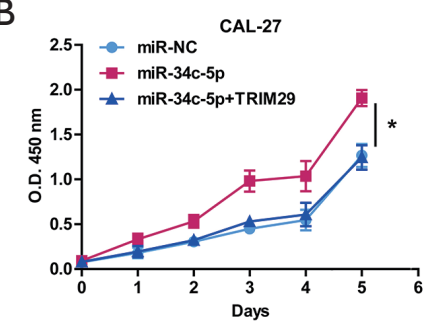

D
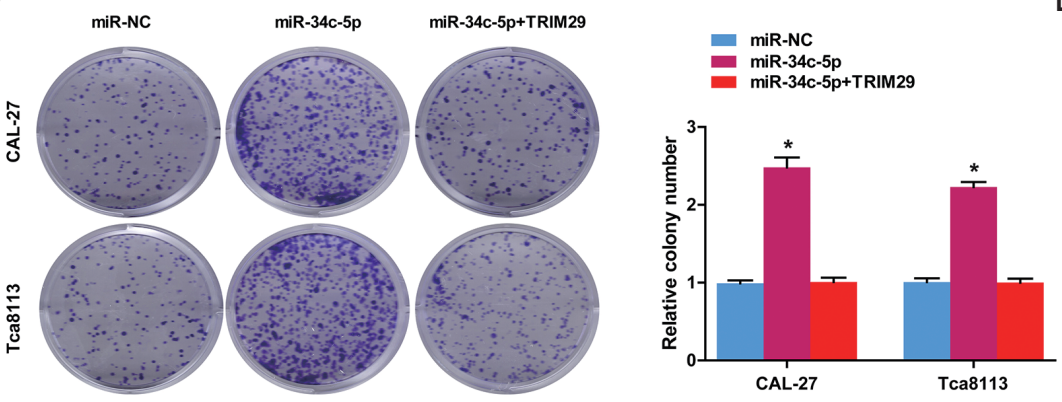

F

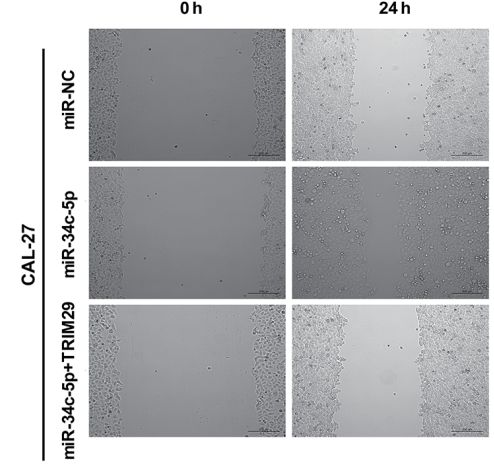

G

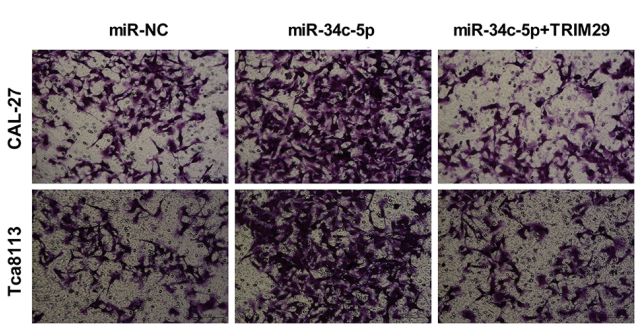

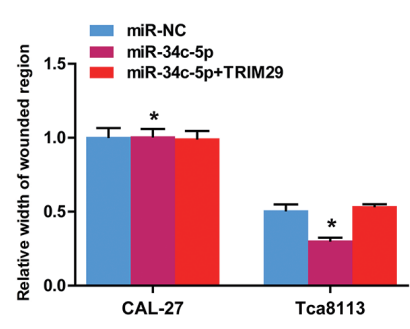

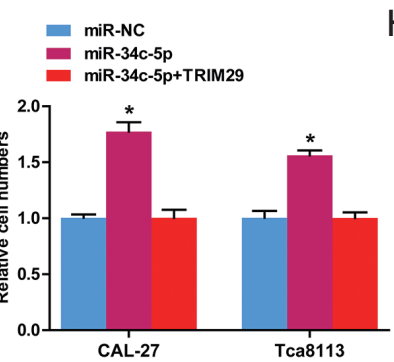

C

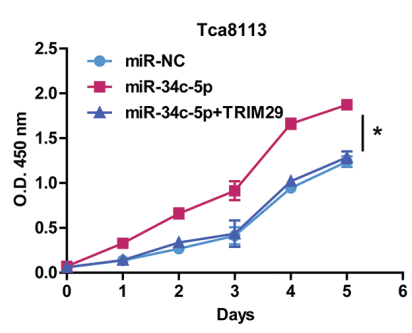

E
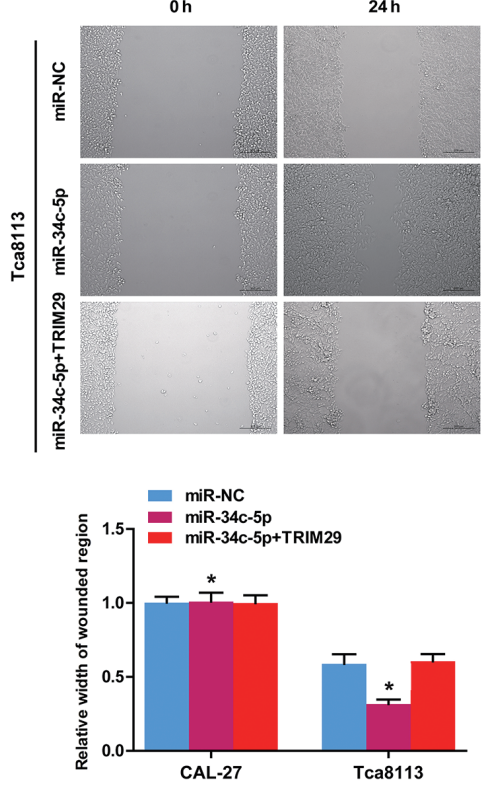

$\mathrm{H}$
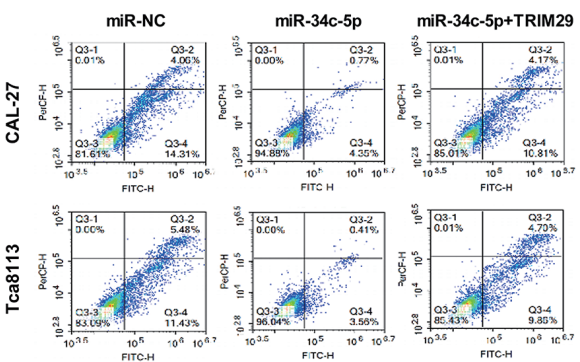

Figure 7 MiR-34c-5p inhibits OSCC cell proliferation, migration, invasion, and apoptosis by regulating TRIM29. (A) Western blot showing the relative protein levels of TRIM29 in CAL-27 and Tca8113 cells transfected with miR-NC, miR-34c-5p, or miR-34c-5p + TRIM29. (B) CCK-8 assay showing cell viability in CAL-27 cells transfected with miR-NC, miR-34c-5p, or miR-34c-5p + TRIM29. (C) CCK-8 assay showing cell viability in Tca8113 cells transfected with miR-NC, miR-34c-5p, or miR-34c-5p + TRIM29. (D) The colony formation test showing cell proliferation in CAL-27 and Tca8113 cells transfected with miR-NC, miR-34c-5p, or miR-34c-5p + TRIM29, a microscope with magnification of $\times 100$. (E) The wound scratch test showing cell migration ability in CAL-27 cells transfected with miRNC, miR-34c-5p, or miR-34c-5p + TRIM29, scale bars $=200 \mu \mathrm{m}$. (F) The wound scratch test showing cell migration ability in Tca8113 cells transfected with miR-NC, miR-34c-5p, or miR-34c-5p + TRIM29, scale bars $=200 \mu \mathrm{m}$. (G) Transwell test showing cell invasion ability in CAL-27 and Tca8113 cells transfected with miR-NC, miR-34c-5p, or miR-34c-5p + TRIM29, scale bars $=200 \mu \mathrm{m}$. *, P<0.05 vs. miRNC group. (H) Flow cytometry showing apoptotic rate in CAL-27 and Tca8113 cells transfected with miR-NC, miR-34c-5p, or miR-34c5p + TRIM29. *, $\mathrm{P}<0.05$ vs. miR-NC group. TRIM29, tripartite motif-containing 29; OSCC, oral squamous cell carcinoma; NC, negative control; CCK-8, cell counting kit-8; OD, optical density. 
closely related to the distant metastasis and pathological grade of OSCC. Importantly, Overexpression of miR$34 c-5 p$ promoted the proliferation, migration, and invasion of CAL-27 and Tca8113 cells, and suppressed their apoptosis. Inversely, low expression of miR-34c-5p suppressed the proliferation, migration, and invasion of CAL-27 and Tca8113 cells, and promoted their apoptosis. This was confirmed in in vivo experiments showing that overexpression of miR-34c-5p promoted tumor growth in the xenograft nude mice model. These results suggested that the expression of miR-34c-5p is closely related to the proliferation, migration, invasion, and apoptosis of OSCC.

TRIM29 is a member of the TRIM protein family. It has multiple zinc finger types and leucine zipper types which can participate in the formation of homodimers or heterodimers combined with nucleic acids. Therefore, it can be used as a transcriptional regulator in the process of carcinogenesis and differentiation $(37,38)$. It has been confirmed that TRIM29 can exert carcinogenic effects in a variety of tumors, such as gastric cancer, small cell lung cancer, pancreatic cancer, and colorectal cancer $(18,22,39,40)$. However, in breast cancer and prostate cancer, TRIM29 expression tends to be lower $(41,42)$. Therefore, the role of TRIM29 in cancer is polymorphic. In fact, this phenomenon is very common because of genetic polymorphism and tumor complexity. This current investigation revealed that TRIM29 is lowly expressed in OSCC tissues and its expression is closely related to the distant metastasis and pathological grade of OSCC. Patients with low levels of TRIM29 expression had a significantly lower disease-free survival rate compared to patients with high TRIM29 expression. Importantly, overexpression of TRIM29 in CAL-27 and Tca8113 cells inhibited proliferation, migration and invasion, and indued their apoptosis. Furthermore, silencing TRIM29 expression promoted cell proliferation, migration invasion, and inhibited apoptosis of OSCC. These results suggested that the expression level of TRIM29 is closely related to the proliferation, invasion, and migration of OSCC.

Tumor metastasis is a complex multi-step process involving tumor cell dissociation from the origin, degradation of the extracellular matrix, cell metastasis and growth, escape from apoptosis, angiogenesis, invasion of surrounding tissues, and cell metastasis to distant parts of the body (43). Current research shows that targeted therapy plays an important role in the treatment of patients with OSCC (44-46). As different miRNAs can behave very differently depending on the tumor type, it is increasingly important to study the targets of individual miRNAs.
Bioinformatic analyses (starBase 2.0) were used to predict the target relationship between miR-34c-5p and TRIM29. CAL-27 cells co-transfected with the wt TRIM29 3'-UTR and miR-34c-5p showed significantly reduced luciferase activity compared to cells transfected with miR-NC. These results suggested that miR-34c-5p binds to TRIM29 to inhibit TRIM29 expression. Importantly, miR-34c-5p regulated OSCC cell proliferation, migration, invasion and apoptosis by inhibiting TRIM29 expression.

This interaction between miR-34c-5p and TRIM29 may be a novel molecular pathway for the pathogenesis of OSCC and may represent potential therapeutic targets for the treatment of OSCC and other malignant tumors.

\section{Acknowledgments}

Funding: This work was supported by the scientific research and innovation fund of the First Affiliated Hospital of Harbin Medical University (2018L004; 2020M13).

\section{Footnote}

Reporting Checklist: The authors have completed the ARRIVE reporting checklist. Available at https://dx.doi. org/10.21037/atm-21-4679

Data Sharing Statement: Available at https://dx.doi. org/10.21037/atm-21-4679

Conflicts of Interest: All authors have completed the ICMJE uniform disclosure form (available at https://dx.doi. org/10.21037/atm-21-4679). The authors have no conflicts of interest to declare.

Ethical Statement: The authors are accountable for all aspects of the work in ensuring that questions related to the accuracy or integrity of any part of the work are appropriately investigated and resolved. The study was conducted in accordance with the Declaration of Helsinki (as revised in 2013). All animal experiments were performed according to the National Institute of Health (NIH) Guide for the Care and Use of Laboratory Animals and were approved by the ethics committee of the First Affiliated Hospital of Harbin Medical University.

Open Access Statement: This is an Open Access article distributed in accordance with the Creative Commons Attribution-NonCommercial-NoDerivs 4.0 International 


\section{Page 16 of 17}

License (CC BY-NC-ND 4.0), which permits the noncommercial replication and distribution of the article with the strict proviso that no changes or edits are made and the original work is properly cited (including links to both the formal publication through the relevant DOI and the license). See: https://creativecommons.org/licenses/by-nc-nd/4.0/.

\section{References}

1. Bray F, Ferlay J, Soerjomataram I, et al. Global cancer statistics 2018: GLOBOCAN estimates of incidence and mortality worldwide for 36 cancers in 185 countries. CA Cancer J Clin 2018;68:394-424.

2. Rivera C. Essentials of oral cancer. Int J Clin Exp Pathol 2015;8:11884-94.

3. Leemans CR, Braakhuis BJ, Brakenhoff RH. The molecular biology of head and neck cancer. Nat Rev Cancer 2011;11:9-22.

4. Amin MB, Greene FL, Edge SB, et al. The Eighth Edition AJCC Cancer Staging Manual: Continuing to build a bridge from a population-based to a more "personalized" approach to cancer staging. CA Cancer J Clin 2017;67:93-9.

5. Hamada T, Wakamatsu T, Miyahara M, et al. MUC4: a novel prognostic factor of oral squamous cell carcinoma. Int J Cancer 2012;130:1768-76.

6. Morandi L, Gissi D, Tarsitano A, et al. DNA methylation analysis by bisulfite next-generation sequencing for early detection of oral squamous cell carcinoma and high-grade squamous intraepithelial lesion from oral brushing. J Craniomaxillofac Surg 2015;43:1494-500.

7. Huang SF, Wei FC, Liao CT, et al. Risk stratification in oral cavity squamous cell carcinoma by preoperative CRP and SCC antigen levels. Ann Surg Oncol 2012;19:3856-64.

8. Kono M, Watanabe M, Abukawa $\mathrm{H}$, et al. Cyclooxygenase-2 expression is associated with vascular endothelial growth factor $\mathrm{C}$ expression and lymph node metastasis in oral squamous cell carcinoma. J Oral Maxillofac Surg 2013;71:1694-702.

9. Sagheb K, Blatt S, Rahimi-Nedjat RK, et al. Cervical metastases behavior of T1-2 squamous cell cancer of the oral mucosa. Clin Oral Investig 2017;21:931-5.

10. Herrera-Espejo S, Santos-Zorrozua B, Álvarez-González P, et al. A Systematic Review of MicroRNA Expression as Biomarker of Late-Onset Alzheimer's Disease. Mol Neurobiol 2019;56:8376-91.

11. Rupaimoole R, Slack FJ. MicroRNA therapeutics: towards a new era for the management of cancer and other diseases.
Shen et al. The role of $\mathrm{miR}-34 \mathrm{c}-5 \mathrm{p}$ in oral squamous cell carcinoma

Nat Rev Drug Discov 2017;16:203-22.

12. Sun LP, Xu K, Cui J, et al. Cancer-associated fibroblast-derived exosomal miR-382-5p promotes the migration and invasion of oral squamous cell carcinoma. Oncol Rep 2019;42:1319-28.

13. Wu Y, Ni Z, Yan X, et al. Targeting the MIR34C-5pATG4B-autophagy axis enhances the sensitivity of cervical cancer cells to pirarubicin. Autophagy 2016;12:1105-17.

14. Amaral AJ, Andrade J, Foxall RB, et al. miRNA profiling of human naive CD4 T cells links miR-34c-5p to cell activation and HIV replication. EMBO J 2017;36:346-60.

15. Gu J, Wang G, Liu H, et al. SATB2 targeted by methylated miR-34c-5p suppresses proliferation and metastasis attenuating the epithelial-mesenchymal transition in colorectal cancer. Cell Prolif 2018;51:e12455.

16. $\mathrm{Xu} \mathrm{W}$, Chen B, Ke D, et al. TRIM29 mediates lung squamous cell carcinoma cell metastasis by regulating autophagic degradation of E-cadherin. Aging (Albany NY) 2020;12:13488-501.

17. Xu R, Hu J, Zhang T, et al. TRIM29 overexpression is associated with poor prognosis and promotes tumor progression by activating $\mathrm{Wnt} / \beta$-catenin pathway in cervical cancer. Oncotarget 2016;7:28579-91.

18. Sun H, Dai X, Han B. TRIM29 as a novel biomarker in pancreatic adenocarcinoma. Dis Markers 2014;2014:317817.

19. Jiang T, Tang HM, Lu S, et al. Up-regulation of tripartite motif-containing 29 promotes cancer cell proliferation and predicts poor survival in colorectal cancer. Med Oncol 2013;30:715.

20. Xu M, Hu J, Zhou B, et al. TRIM29 prevents hepatocellular carcinoma progression by inhibiting Wnt/ $\beta$-catenin signaling pathway. Acta Biochim Biophys Sin (Shanghai) 2019;51:68-77.

21. Ai L, Kim WJ, Alpay M, et al. TRIM29 suppresses TWIST1 and invasive breast cancer behavior. Cancer Res 2014;74:4875-87.

22. Kosaka Y, Inoue H, Ohmachi T, et al. Tripartite motifcontaining 29 (TRIM29) is a novel marker for lymph node metastasis in gastric cancer. Ann Surg Oncol 2007;14:2543-9.

23. Yanagi T, Watanabe M, Hata H, et al. Loss of TRIM29 Alters Keratin Distribution to Promote Cell Invasion in Squamous Cell Carcinoma. Cancer Res 2018;78:6795-806.

24. Keller A, Leidinger P, Bauer A, et al. Toward the bloodborne miRNome of human diseases. Nat Methods 2011;8:841-3.

25. Keller A, Leidinger P, Vogel B, et al. miRNAs can be 
generally associated with human pathologies as exemplified for miR-144. BMC Med 2014;12:224.

26. Kong L, Liu P, Zheng M, et al. The miR-1224-5p/ELF3 Axis Regulates Malignant Behaviors of Pancreatic Cancer via PI3K/AKT/Notch Signaling Pathways. Onco Targets Ther 2020;13:3449-66.

27. Subramanian A, Tamayo P, Mootha VK, et al. Gene set enrichment analysis: a knowledge-based approach for interpreting genome-wide expression profiles. Proc Natl Acad Sci U S A 2005;102:15545-50.

28. Li JH, Liu S, Zhou H, et al. starBase v2.0: decoding miRNA-ceRNA, miRNA-ncRNA and protein-RNA interaction networks from large-scale CLIP-Seq data. Nucleic Acids Res 2014;42:D92-7.

29. Yang JH, Li JH, Shao P, et al. starBase: a database for exploring microRNA-mRNA interaction maps from Argonaute CLIP-Seq and Degradome-Seq data. Nucleic Acids Res 2011;39:D202-9.

30. Ji Q, Xu X, Xu Y, et al. miR-105/Runx2 axis mediates FGF2-induced ADAMTS expression in osteoarthritis cartilage. J Mol Med (Berl) 2016;94:681-94.

31. Hanahan D, Weinberg RA. Hallmarks of cancer: the next generation. Cell 2011;144:646-74.

32. Zhang B, Li Y, Hou D, et al. MicroRNA-375 Inhibits Growth and Enhances Radiosensitivity in Oral Squamous Cell Carcinoma by Targeting Insulin Like Growth Factor 1 Receptor. Cell Physiol Biochem 2017;42:2105-17.

33. Wang K, Jin J, Ma T, et al. MiR-139-5p inhibits the tumorigenesis and progression of oral squamous carcinoma cells by targeting HOXA9. J Cell Mol Med 2017;21:3730-40.

34. Wang Y, Wang X, Tang J, et al. The study of mechanism of miR-34c-5p targeting FLOT2 to regulate proliferation, migration and invasion of osteosarcoma cells. Artif Cells Nanomed Biotechnol 2019;47:3559-68.

35. Wei H, Wang X, Niu X, et al. miR-34c-5p targets Notch1 and suppresses the metastasis and invasion of cervical cancer. Mol Med Rep 2021;23:120.

36. Li N, Mao D, Cao Y, et al. Downregulation of SIRT6 by $\mathrm{miR}-34 \mathrm{c}-5 \mathrm{p}$ is associated with poor prognosis and promotes colon cancer proliferation through inhibiting apoptosis via the JAK2/STAT3 signaling pathway. Int J Oncol 2018;52:1515-27.

37. Hatakeyama S. TRIM proteins and cancer. Nat Rev Cancer 2011;11:792-804.

38. Napolitano LM, Meroni G. TRIM family: Pleiotropy and diversification through homomultimer and heteromultimer formation. IUBMB Life 2012;64:64-71.

39. Tang ZP, Dong QZ, Cui QZ, et al. Ataxia-telangiectasia group D complementing gene (ATDC) promotes lung cancer cell proliferation by activating $\mathrm{NF}-\kappa \mathrm{B}$ pathway. PLoS One 2013;8:e63676.

40. Wang L, Heidt DG, Lee CJ, et al. Oncogenic function of ATDC in pancreatic cancer through Wnt pathway activation and beta-catenin stabilization. Cancer Cell 2009;15:207-19.

41. Ernst T, Hergenhahn M, Kenzelmann M, et al. Decrease and gain of gene expression are equally discriminatory markers for prostate carcinoma: a gene expression analysis on total and microdissected prostate tissue. Am J Pathol 2002;160:2169-80.

42. Nacht M, Ferguson AT, Zhang W, et al. Combining serial analysis of gene expression and array technologies to identify genes differentially expressed in breast cancer. Cancer Res 1999;59:5464-70.

43. Cao H, Xu E, Liu H, et al. Epithelial-mesenchymal transition in colorectal cancer metastasis: A system review. Pathol Res Pract 2015;211:557-69.

44. Huang WC, Jang TH, Tung SL, et al. A novel miR-365$3 \mathrm{p} / \mathrm{EHF} /$ keratin 16 axis promotes oral squamous cell carcinoma metastasis, cancer stemness and drug resistance via enhancing $\beta 5$-integrin/c-met signaling pathway. J Exp Clin Cancer Res 2019;38:89.

45. Sun S, Wang J, Liu J, et al. MiR-302b Suppresses Tumor Metastasis by Targeting Frizzled 6 in OSCC. J Dent Res 2021;100:739-45.

46. Kong D, Zhang G, Ma H, et al. miR-1271 inhibits OSCC cell growth and metastasis by targeting ALK. Neoplasma 2015;62:559-66.

(English Language Editor: J. Teoh)
Cite this article as: Shen Y, Sun C, Zhao B, Guo H, Li J, Xia Y, Liu M, Piao S, Saiyin W. miR-34c-5p mediates the cellular malignant behaviors of oral squamous cell carcinoma through targeted binding of TRIM29. Ann Transl Med 2021;9(20):1537. doi: 10.21037/atm-21-4679 